\title{
Invariance Control with Time-varying Constraints
}

\author{
Melanie Kimmel and Sandra Hirche
}

\begin{abstract}
In general, dynamic systems have to meet certain requirements in order to achieve a prescribed performance or to ensure safety. These constraints on the states and/or outputs of the system may even be changing over time. This generates the necessity for a control scheme, which is able to enforce time-varying constraints. In this work, we propose a control approach, which uses the concepts of invariance control to enforce constraints with time-varying parameters. A control law is derived, which guarantees the satisfaction of constraints with bounded time-varying parameters. Stability properties are investigated and illustrated in a numerical example.
\end{abstract}

\section{INTRODUCTION}

In engineering applications, constraints are often imposed on the system behavior to meet performance goals and/or to ensure the safe operation. The frequency of electrical energy transmission systems, for example, has to stay within a predefined range, an autonomous car is expected to stay on the road and drive without accidents and an industrial robot should not injure any humans in its vicinity. Although the applications differ, in each case, there is a necessity to impose safety or performance bounds on the states and/or outputs of the particular system. These bounds are usually enforced by means of control and as the systems are in general located in a dynamic environment, the control scheme has to be able to process time-varying constraints.

Probably the most well-known control scheme in such a setup is model predictive control (MPC) [1]. It is an optimization-based approach, which enforces input, state and output constraints. For high dimensional, nonlinear, dynamic systems, however, the computationally expensive optimization process may prevent the real-time application. Input and state constraints on systems with disturbances may be enforced by the reference governor approach [2], but similarly to MPC, real-time requirements might be violated due to the required numerical simulation. Another method for verifying guaranteed constraint enforcement is provided by barrier certificates [3], which build the base for the development of constraint enforcing control schemes [4]. However, as the barrier functions are designed to approach an infinite value on approaching a constraint, they do not provide a defined behavior if constraint violation occurs, e.g. due to an initial violation or a change in the set of constraints. A promising control scheme to realize safety or performance bounds on states and outputs is invariance control, which is similar to the previously mentioned control based on barrier

All authors are with the Chair of Information-Oriented Control, Department of Electrical Engineering and Information Technology, Technical University of Munich, D-80290 München, Germany. \{melanie.kimmel, hirche\}@tum.de certificates. Instead of using a barrier function, however, the system dynamics and the boundary information are combined in a so-called invariance function, which determines the necessity of corrective action and has a well-defined value even for violated constraints. The special choice of the invariance function allows the control design as an add-on to a working, stabilizing control structure [5], while ensuring that multiple state and output constraints are not violated [6]. However, so far, only static constraints are provably enforced with the invariance control approach for general nonlinear, control affine systems.

In this work, we introduce an invariance control approach, which enforces time-varying constraints and is applicable to control affine, nonlinear MIMO systems. With a design as an add-on to an existing, stabilizing controller, it is useful for a wide range of applications. It enables a desired behavior whenever possible but at the same time enforces time-varying boundaries. The stability of the proposed control scheme is shown using Lyapunov theory. Furthermore, conditions are derived under which the constrained system is controlled positively invariant. A numerical example illustrates the characteristics of the approach.

The remainder of this paper is organized as follows: Section II introduces the invariance control design for systems with time-varying constraints. In Section III and IV, stability and invariance properties are discussed, respectively. Section V provides an illustrative numerical example.

Notation: Vectors and matrices are denoted by bold, small and capital characters, respectively. The Euclidean vector norm (2-norm) is given by $\|\boldsymbol{x}\|_{2}=\sqrt{\boldsymbol{x}^{\top} \boldsymbol{x}}$ for $\boldsymbol{x} \in \mathbb{R}^{n}$, while the element-wise inequality of $\boldsymbol{x}_{1}, \boldsymbol{x}_{2} \in \mathbb{R}^{n}$ is abbreviated by $\boldsymbol{x}_{1} \preccurlyeq \boldsymbol{x}_{2}$. The $k$ row vectors $\boldsymbol{a}_{1}^{\top}, \ldots, \boldsymbol{a}_{k}^{\top} \in \mathbb{R}^{1 \times n}$ and scalars $b_{1}, \ldots, b_{k} \in \mathbb{R}$ are stacked to yield matrix $\boldsymbol{A}=$ $\left[\boldsymbol{a}_{i}^{\boldsymbol{\top}}\right] \in \mathbb{R}^{k \times n}$ and vector $\boldsymbol{b}=\left[b_{i}\right] \in \mathbb{R}^{k \times 1}$ with

$$
\boldsymbol{A}=\left[\boldsymbol{a}_{i}^{\top}\right]=\left[\begin{array}{c}
\boldsymbol{a}_{1}^{\top} \\
\vdots \\
\boldsymbol{a}_{k}^{\top}
\end{array}\right], \quad \boldsymbol{b}=\left[\boldsymbol{b}_{i}\right]=\left[\begin{array}{c}
b_{1} \\
\vdots \\
b_{k}
\end{array}\right],
$$

respectively. Time derivatives are represented by dots $\dot{x}=\frac{\mathrm{d} x}{\mathrm{~d} t}$ or, for higher orders, by $x^{(i)}=\frac{\mathrm{d}^{i} x}{\mathrm{~d} t^{i}}$. The expression

$$
\mathcal{L}_{\boldsymbol{f}} h(\boldsymbol{x})=\frac{\partial h}{\partial \boldsymbol{x}} \boldsymbol{f}
$$

denotes the directional derivative of a function $h: \mathbb{R}^{n} \rightarrow \mathbb{R}$ in direction $\boldsymbol{f}$, i.e. the first order Lie-derivative. $\mathcal{L}_{\boldsymbol{f}}^{i} h(\boldsymbol{x})$, the Lie-derivative of order $i$, is defined recursively. The set of $k$ times continuously differentiable functions $\boldsymbol{h}: \mathbb{R}^{n} \rightarrow \mathbb{R}^{m}$ is denoted by $\mathcal{C}^{k}$. 


\section{INVARIANCE CONTROL WITH TIME-VARYING CONSTRAINTS}

Invariance control for systems with static boundaries is introduced in [5]-[7]. This section provides the design steps for invariance control with time-varying constraints.

The implementation of an invariance controller as an add-on to an existing controller enforces the adherence to output and/or state constraints, while a desired control goal is pursued whenever possible [5]. The schematic structure of the controlled system is depicted in Fig. 1. The nominal

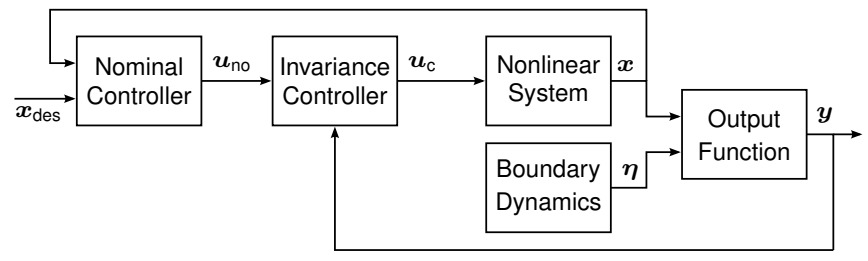

Fig. 1: Structure of an invariance controlled system with time-varying boundaries

controller generates a nominal control input $\boldsymbol{u}_{\text {no }}$ from the system outputs/states and corresponding desired values. The invariance controller processes the nominal control input and the constraint information, given by an output function, to determine a corrective control input $\boldsymbol{u}_{\mathrm{c}}$, which is as close as possible to the nominal control input while ensuring adherence to the constraints. So far, these output functions may only depend on the states and/or outputs of the system and constant parameters $\boldsymbol{\eta}$. In this work, we allow output functions, which depend on system states/outputs as well as on time-varying parameters $\boldsymbol{\eta}(t)$. Invariance control is applicable to nonlinear, control affine MIMO systems

$$
\left.\begin{array}{l}
\dot{\boldsymbol{x}}=\boldsymbol{f}(\boldsymbol{x})+\boldsymbol{G}(\boldsymbol{x}) \boldsymbol{u} \\
\boldsymbol{y}_{\text {out }}=\boldsymbol{h}_{\text {out }}(\boldsymbol{x})
\end{array}\right\}
$$

with the states $\boldsymbol{x} \in \mathbb{R}^{n}$, the input $\boldsymbol{u} \in \mathcal{U}_{\text {no }} \subset \mathbb{R}^{m}$, and sufficiently smooth functions $\boldsymbol{f}: \mathbb{R}^{n} \rightarrow \mathbb{R}^{n}, \boldsymbol{g}_{i}: \mathbb{R}^{n} \rightarrow \mathbb{R}^{n}$ with $\boldsymbol{G}=\left[\boldsymbol{g}_{1} \ldots \boldsymbol{g}_{m}\right]$, cf. [6]. The natural system output is given by $\boldsymbol{h}_{\text {out }}: \mathbb{R}^{n} \rightarrow \mathbb{R}^{q}$. The following design steps are also valid for time-invariant constraints and single input and/or single output systems if not indicated differently.

\section{A. Output Functions}

The set of $l$ constraints, with $l$ being arbitrarily large [8], defines a vector of time-varying, continuous output functions

$$
\boldsymbol{y}=\boldsymbol{h}(\boldsymbol{x}, \boldsymbol{\eta}(t)),
$$

with $\boldsymbol{y} \in \mathbb{R}^{l}$ and the parameter vector $\boldsymbol{\eta}(t) \in \mathbb{R}^{n_{\eta}}$. Each element $y_{i}=h_{i}(\boldsymbol{x}, \boldsymbol{\eta}(t))$ describes one constraint $i$.

We consider bounded parameters, which are sufficiently often continuously differentiable with respect to time.

Assumption 1: The time-varying parameter vector $\boldsymbol{\eta}(t)$ is given by a bounded $\mathcal{C}^{r_{i}}$ function with bounded derivatives

$$
\boldsymbol{\eta}^{(j)}(t) \in\left[\boldsymbol{\eta}_{\min }^{(j)}, \boldsymbol{\eta}_{\text {max }}^{(j)}\right] \quad \forall 0 \leq j \leq r_{i} .
$$

Each output function is equal to zero right on the constraint and negative in the time-varying admissible set

$$
\mathcal{H}(t)=\left\{\boldsymbol{x} \in \mathbb{R}^{n} \mid h_{i}(\boldsymbol{x}, \boldsymbol{\eta}(t)) \leq 0 \quad \forall 1 \leq i \leq l\right\} .
$$

Invariance control is applied to keep the system within the admissible set, which is only possible if this set exists.

Assumption 2: The admissible set $\mathcal{H}(t)$ (4) is never empty

$$
\mathcal{H}(t) \neq \varnothing \quad \forall t \geq 0
$$

The output functions provide a measure for the distance of the state to the constraints. In order to keep the state within the admissible set, it is, however, not enough to monitor the output function. The change of the output function over time, the influence of the control input and the constraint dynamics have to be considered as well. Therefore, the system is inputoutput linearized with respect to the output functions (2).

\section{B. Input-Output (I/O)-Linearization}

As the output functions depend on the time-varying parameters $\boldsymbol{\eta}(t)$, I/O-linearization for time-varying systems [9] is applied. The I/O-linearization of a time-varying system with $n$ states with respect to an output $h_{i}(\boldsymbol{x}, \boldsymbol{\eta}(t))$ is represented by a time-varying invertible coordinate transformation

$$
\left(\begin{array}{c}
\boldsymbol{\xi}_{\text {int }, i} \\
\boldsymbol{\xi}_{i}
\end{array}\right)=T_{i}(\boldsymbol{x}, t)=\left(\begin{array}{c}
\varphi_{1}(\boldsymbol{x}, t) \\
\vdots \\
\varphi_{n-r_{i}}(\boldsymbol{x}, t) \\
h_{i}(\boldsymbol{x}, \boldsymbol{\eta}(t)) \\
\overline{\mathcal{L}}_{f}^{1} h_{i}(\boldsymbol{x}, \boldsymbol{\eta}(t)) \\
\vdots \\
\overline{\mathcal{L}}_{f}^{r_{i}-1} h_{i}(\boldsymbol{x}, \boldsymbol{\eta}(t))
\end{array}\right),
$$

where $\boldsymbol{\xi}_{i} \in \mathbb{R}^{r_{i}}$ are the states of the integrator chain resulting from the linearization and $\boldsymbol{\xi}_{\text {int }, i} \in \mathbb{R}^{n-r_{i}}$ are the states of the internal dynamics, generally assuming $r_{i}<n$. The transformations $\varphi_{j}(\boldsymbol{x}, t)$ determine the states of the internal dynamics. As stated in [9], the internal states have no influence on the output, which means they fulfill

$$
\left(\frac{\partial}{\partial \boldsymbol{x}}\left(\begin{array}{c}
\varphi_{1}(\boldsymbol{x}, t) \\
\vdots \\
\varphi_{n-r_{i}}(\boldsymbol{x}, t)
\end{array}\right)\right) \boldsymbol{G}(\boldsymbol{x})=\frac{\partial \boldsymbol{\varphi}(\boldsymbol{x}, t)}{\partial \boldsymbol{x}} \boldsymbol{G}(\boldsymbol{x})=\mathbf{0} .
$$

We consider the time-invariant system (1) with the timevarying output function (2). Therefore, the input transformation of the I/O-linearization is given by [10]

$$
z_{i}=y_{i}^{\left(r_{i}\right)}=\boldsymbol{a}_{i}^{\top}(\boldsymbol{x}, \boldsymbol{\eta}(t)) \boldsymbol{u}+b_{i}\left(\boldsymbol{x}, \boldsymbol{\eta}(t), \ldots, \boldsymbol{\eta}^{\left(r_{i}\right)}(t)\right)
$$

with the relative degree $r_{i}$, the pseudo input $z_{i}$,

$$
\begin{aligned}
& \boldsymbol{a}_{i}^{\top}(\boldsymbol{x}, \boldsymbol{\eta}(t))=\left[\mathcal{L}_{\boldsymbol{g}_{1}} \mathcal{L}_{\boldsymbol{f}}^{r_{i}-1} y_{i} \ldots \mathcal{L}_{\boldsymbol{g}_{m}} \mathcal{L}_{\boldsymbol{f}}^{r_{i}-1} y_{i}\right], \\
& \boldsymbol{a}_{i}^{\boldsymbol{\top}}(\boldsymbol{x}, \boldsymbol{\eta}(t)) \neq \mathbf{0}^{T}, \\
& {\left[\mathcal{L}_{\boldsymbol{g}_{1}} \mathcal{L}_{\boldsymbol{f}}^{r-1} y_{i} \ldots \mathcal{L}_{\boldsymbol{g}_{m}} \mathcal{L}_{\boldsymbol{f}}^{r-1} y_{i}\right]=\mathbf{0}^{T} \quad \forall r<r_{i},} \\
& b_{i}\left(\boldsymbol{x}, \boldsymbol{\eta}(t), \ldots, \boldsymbol{\eta}^{\left(r_{i}\right)}(t)\right)=\overline{\mathcal{L}}_{\boldsymbol{f}}^{r_{i}} y_{i},
\end{aligned}
$$

and the time-dependent Lie operator defined as

$$
\overline{\mathcal{L}}_{\boldsymbol{f}}^{r_{i}} y_{i}=\left(\frac{\partial}{\partial t}+\mathcal{L}_{\boldsymbol{f}}\right)^{r_{i}} y_{i} .
$$


Note that, due to the combination of the time-invariant state dynamics with the time-varying output functions, the timedependent Lie operator is only required for determining the vector $b_{i}\left(\boldsymbol{x}, \boldsymbol{\eta}(t), \ldots, \boldsymbol{\eta}^{\left(r_{i}\right)}(t)\right)$. The I/O-linearization is only valid, if the relative degree $r_{i}$ is well-defined, which is assured if the following assumptions hold [10].

Assumption 3: Each element of the output function (2) is a $\mathcal{C}^{r_{i}}$ function with respect to time .

Assumption 4: The vector $\boldsymbol{a}_{i}^{\top}(\boldsymbol{x}, \boldsymbol{\eta}(t))$ has at least one non-zero element, i.e. $\boldsymbol{a}_{i}^{\top}(\boldsymbol{x}, \boldsymbol{\eta}(t)) \neq \mathbf{0} \forall t \geq 0$.

Both assumptions are not very restrictive since they are assured by a sensible choice of constraints. As the system is, in general, subject to multiple constraints, the I/O-linearization also requires a well-defined vector relative degree [11].

Definition 1: I/O-linearization of (1) w.r.t. the output (2) yields a well-defined vector relative degree $\left(r_{1}, \ldots, r_{l}\right)$ on a subset $\mathcal{X}$ of the state space if for all $\boldsymbol{x} \in \mathcal{X} \subseteq \mathbb{R}^{n}$

1) each constraint $1 \leq i \leq l$ fulfills (8)-(10) and

2) the decoupling matrix has full row rank.

$$
\operatorname{det}(\boldsymbol{A}(\boldsymbol{x}))=\operatorname{det}\left(\left[\begin{array}{c}
\boldsymbol{a}_{1}^{\top}(\boldsymbol{x}, \boldsymbol{\eta}(t)) \\
\vdots \\
\boldsymbol{a}_{l}^{\top}(\boldsymbol{x}, \boldsymbol{\eta}(t))
\end{array}\right]\right)=l
$$

Naturally, for $l \geq m$ the second condition will never be fulfilled. How this issue is resolved by the way corrective control is determined will be discussed briefly in the corresponding section.

\section{Invariance Functions}

Combining the output functions with the dynamics of the system (1) leads to the concept of invariance functions $\Phi_{i}\left(\boldsymbol{x}, t, \gamma_{i}\right)$. They determine, whether corrective control action is necessary. The derivation is based on the results of the I/O-linearization (7). For each constraint $i$, the I/Olinearization yields an integrator chain with the output $y_{i}$, the input $z_{i}$ and $r_{i}$ states [5]. The states are determined by the mapping $\boldsymbol{\xi}_{i}\left(\boldsymbol{x}, \boldsymbol{\eta}(t), \ldots, \boldsymbol{\eta}^{\left(r_{i}-1\right)}(t)\right)=\left[y_{i} \ldots y_{i}^{\left(r_{i}-1\right)}\right]^{\top}$.

For a constant input $z_{i}=\gamma_{i}$, the output is determined by integrating $r_{i}$ times with respect to time

$$
y_{i}(t)=p_{i}\left(t, \boldsymbol{\xi}_{i, 0}, \gamma_{i}\right)=\frac{t^{r_{i}}}{r_{i} !} \gamma_{i}+\sum_{k=0}^{r_{i}-1} \frac{t^{k}}{k !} y_{i, 0}^{(k)},
$$

where $\boldsymbol{\xi}_{i, 0}=\left[y_{i, 0} \ldots y_{i, 0}^{\left(r_{i}-1\right)}\right]^{\top}$ denote the values at $t=t_{0}$. Calculating the maximum of (13) for all future times

$$
\max _{\tau>t_{0}} p_{i}\left(\tau, \boldsymbol{\xi}_{i, 0}, \gamma_{i}\right)
$$

yields a measure for the minimal distance of the state to the constraint if an input $z_{i}=\gamma_{i}<0$ is applied at $t=t_{0}$. The state stays within the admissible set (4) if the maximum is negative. These considerations yield the invariance function for time-varying constraints

$$
\Phi_{i}\left(\boldsymbol{x}, t, \gamma_{i}\right)=\max _{\tau>t} p_{i}\left(\tau, \boldsymbol{\xi}_{i}\left(\boldsymbol{x}, \boldsymbol{\eta}(t), \ldots, \boldsymbol{\eta}^{\left(r_{i}-1\right)}(t)\right), \gamma_{i}\right)
$$

with (13) and the parameter $\gamma_{i}<0$.

The invariance function determines the maximum value of the output function if a counteracting input $z_{i}=\gamma_{i}<0$ is applied to the integrator chain at time $t$. A non-negative value indicates a possible constraint violation in the future and the necessity of corrective control action to avoid a violation of the corresponding constraint. It also defines the invariant set

$$
\mathcal{G}\left(t, \gamma_{i}\right)=\left\{\boldsymbol{x} \in \mathbb{R}^{n} \mid \Phi_{i}\left(\boldsymbol{x}, t, \gamma_{i}\right) \leq 0 \quad \forall 1 \leq i \leq l\right\}
$$

The corrective control input $\boldsymbol{u}_{\mathrm{c}}$ of the invariance controller has to be determined such that it renders the nonlinear system (1) controlled positive invariant with respect to the set (15). Then, the system states remain within the invariant set and as a result also within the admissible set (4) for all future times, once the set is entered [5].

For notational convenience, we will omit the explicit timedependencies $\boldsymbol{\eta}(t) \ldots \boldsymbol{\eta}^{\left(r_{i}\right)}(t)$ in the following.

\section{Corrective Control}

Constraints with a negative invariance function are not in danger of being violated, i.e. for these constraints, nominal control suffices. The remaining constraints with a nonnegative $\Phi_{i}\left(\boldsymbol{x}, t, \gamma_{i}\right)$ are called active [6] and require corrective control. They are collected in the set of active constraints

$$
\mathcal{K}=\left\{i \in\{1,2, \ldots, l\} \mid \Phi_{i}\left(\boldsymbol{x}, \boldsymbol{\eta}, \ldots, \boldsymbol{\eta}^{\left(r_{i}-1\right)}, \gamma_{i}\right) \geq 0\right\} .
$$

Positive invariance with respect to (15) is achieved, if either the system motion is directed away from the constraints

$$
y_{i}^{(r)}<0 \quad \forall 1 \leq r \leq r_{i}-1
$$

or an appropriate input is provided, which fulfills

$$
y_{i}^{\left(r_{i}\right)} \leq \gamma_{i}<0
$$

i.e. $\gamma_{i}<0$ is an upper bound on the derivative $y_{i}^{\left(r_{i}\right)}$. Choosing the pseudo input (7) such that $z_{i} \leq \gamma_{i}<0$ holds for active constraints, fulfills (18) and results in positive invariance of the integrator chain [5]. This yields an elementwise condition on the control input $\boldsymbol{u}$

$$
\boldsymbol{A}_{\mathcal{K}}(\boldsymbol{x}, \boldsymbol{\eta}) \boldsymbol{u}+\boldsymbol{b}_{\mathcal{K}}\left(\boldsymbol{x}, \boldsymbol{\eta}, \ldots, \boldsymbol{\eta}^{\left(r_{i}\right)}\right) \preccurlyeq \boldsymbol{\gamma} .
$$

with the vector $\boldsymbol{b}_{\mathcal{K}}\left(\boldsymbol{x}, \boldsymbol{\eta}, \ldots, \boldsymbol{\eta}^{\left(r_{i}\right)}\right)=\left[b_{i}\left(\boldsymbol{x}, \boldsymbol{\eta}, \ldots, \boldsymbol{\eta}^{\left(r_{i}\right)}\right)\right]$, the matrix $\boldsymbol{A}_{\mathcal{K}}(\boldsymbol{x}, \boldsymbol{\eta})=\left[\boldsymbol{a}_{i}^{\top}(\boldsymbol{x}, \boldsymbol{\eta})\right]$, the vector $\boldsymbol{\gamma}=\left[\gamma_{i}\right]$ and $i \in \mathcal{K}$. In order to derive a corrective control input which adheres to the constraints and is as close as possible to the nominal control input in the sense of the Euclidean distance, the constrained minimization problem

$$
\begin{gathered}
\boldsymbol{u}_{\mathrm{c}}=\min _{\boldsymbol{u}}\left\|\boldsymbol{u}_{\mathrm{c}}-\boldsymbol{u}_{\mathrm{no}}\right\|_{2}^{2} \\
\text { s.t. } \boldsymbol{A}_{\mathcal{K}}(\boldsymbol{x}, \boldsymbol{\eta}) \boldsymbol{u}+\boldsymbol{b}_{\mathcal{K}}\left(\boldsymbol{x}, \boldsymbol{\eta}, \ldots, \boldsymbol{\eta}^{\left(r_{i}\right)}\right) \preccurlyeq \gamma
\end{gathered}
$$

is solved. Note that it is rather straightforward to include input constraints by adding them as additional conditions to the minimization problem.

As (20) presents a convex optimization with convex constraints, the solution $\boldsymbol{u}_{\mathrm{c}} \in \mathbb{R}^{m}$ is uniquely defined by a limited set of $j \leq m$ constraints with linearly independent $\boldsymbol{a}_{i}^{\top}(\boldsymbol{x}, \boldsymbol{\eta})$ for which the equality holds [12]. These constraints constitute the set $\mathcal{K}_{\mathcal{I}}$ and fulfill

$$
\left.\begin{array}{rl}
\boldsymbol{a}_{i}^{\boldsymbol{\top}}(\boldsymbol{x}, \boldsymbol{\eta}) \boldsymbol{u}_{\mathrm{c}}+b_{i}(\boldsymbol{x}, \boldsymbol{\eta}, \ldots) & =\gamma_{i} \forall i \in \mathcal{K}_{\mathcal{I}} \\
\boldsymbol{a}_{i}^{\boldsymbol{\top}}(\boldsymbol{x}, \boldsymbol{\eta}) \boldsymbol{a}_{j}(\boldsymbol{x}, \boldsymbol{\eta}) & =0 \quad \forall i \neq j \in \mathcal{K}_{\mathcal{I}} .
\end{array}\right\}
$$


The output consisting of the constraints $i \in \mathcal{K}_{\mathcal{I}}$ has a welldefined vector relative degree as it fulfills both conditions in Def. 1. This means that even if an arbitrary number of constraints is defined, Ass. 4 suffices to ensure that the I/Olinearization in the solution remains feasible. If the set $\mathcal{K}_{\mathcal{I}}$ is known, e.g. because $\mathcal{K}$ only contains linearly independent constraints, an explicit analytical solution of (20) is given by

$$
\boldsymbol{u}_{\mathrm{c}}=\boldsymbol{A}_{\mathcal{K}_{\mathcal{I}}}^{+}\left(\boldsymbol{z}_{\mathrm{c}}-\boldsymbol{z}_{\mathrm{no}}\right)+\boldsymbol{u}_{\mathrm{no}}
$$

with $\boldsymbol{z}_{\mathrm{no}}=\boldsymbol{A}_{\mathcal{K}_{\mathcal{I}}}(\boldsymbol{x}, \boldsymbol{\eta}) \boldsymbol{u}_{\mathrm{no}}+\boldsymbol{b}_{\mathcal{K}_{\mathcal{I}}}\left(\boldsymbol{x}, \boldsymbol{\eta}, \ldots, \boldsymbol{\eta}^{\left(r_{i}\right)}\right)$, the Moore-Penrose pseudo inverse $\boldsymbol{A}_{\mathcal{K}_{\mathcal{I}}}^{+}=\boldsymbol{A}_{\mathcal{K}_{\mathcal{I}}}^{\top}\left(\boldsymbol{A}_{\mathcal{K}_{\mathcal{I}}} \boldsymbol{A}_{\mathcal{K}_{\mathcal{I}}}^{\top}\right)^{-1}$ and the corrective pseudo input

$$
z_{\mathrm{c}, i}= \begin{cases}\gamma_{i} & \text { if }\left(z_{\mathrm{no}, i}>\gamma_{i}\right) \wedge\left(\Phi_{i} \geq 0\right) \\ z_{\mathrm{no}, i} & \text { if }\left(z_{\mathrm{no}, i} \leq \gamma_{i}\right) \vee\left(\Phi_{i}<0\right)\end{cases}
$$

It remains to show that the control with time-varying boundaries does not destabilize the system and the system is actually rendered positively invariant.

\section{StABILITy}

Whenever the set of active constraints (16) is empty, nominal control is applied to the system (1). Therefore, the invariance controlled system may only be stable in the sense of Lyapunov, if the nominally controlled system without the invariance controller is stable in the sense of Lyapunov [6].

Assumption 5: The system (1) under nominal control $\boldsymbol{u}_{\text {no }}$ is stable in the sense of Lyapunov.

Since the nominal control law is independent from the choice of constraints on the system as illustrated in Fig. 1, the system under nominal control is obviously also stabilized in the sense of Lyapunov for time-varying boundaries.

The insertion of the invariance controller may destabilize the system, as the internal dynamics resulting from I/Olinearization might be unstable. Therefore, it is necessary to show that the time-varying parameters do not add an additional source of instability. In order to show stability of the internal dynamics with time-varying constraints, we first determine an expression for the coordinate transformation representing the I/O-linearization (5), and especially for the states of the internal dynamics.

Theorem 1: Consider the system (1) and the output function (2). Let Assumptions 3 and 4 hold. Let the transformation representing the I/O-linearization be given by (5). Then there exists a time-invariant mapping $\boldsymbol{\Psi}_{i}(\boldsymbol{x})$ for the states of the internal dynamics such that (5) with $\boldsymbol{\varphi}(\boldsymbol{x}, t)=\boldsymbol{\Psi}_{i}(\boldsymbol{x})$ represents the transformation given by the I/O-linearization.

Proof: We start by analyzing the I/O-linearization with respect to time-invariant constraints, i.e. we assume that the parameters $\boldsymbol{\eta}(t)$ are time-invariant $\boldsymbol{\eta}(t)=\boldsymbol{\eta}$. Then, there exists a time-invariant representation [13]

$$
\left(\begin{array}{c}
\boldsymbol{\xi}_{\text {int }, i} \\
-\boldsymbol{\xi}_{i}
\end{array}\right)=T_{i}(\boldsymbol{x})=\left(\begin{array}{c}
\boldsymbol{\Psi}_{i}(\boldsymbol{x}) \\
h_{i}(\boldsymbol{x}) \\
\mathcal{L}_{f}^{1} h_{i}(\boldsymbol{x}) \\
\vdots \\
\mathcal{L}_{f}^{r_{i}-1} h_{i}(\boldsymbol{x})
\end{array}\right),
$$

of the I/O-linearization of system (1) with respect to the output function $\boldsymbol{h}(\boldsymbol{x}, \boldsymbol{\eta})$ with constant parameters $\left(\frac{d^{k} \boldsymbol{\eta}}{d t^{k}}=\mathbf{0}\right.$

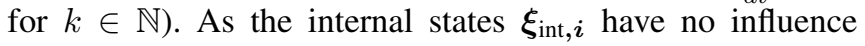
on the system outputs due to the construction of the I/Olinearization [13], their transformation fulfills the condition

$$
\frac{\partial \boldsymbol{\Psi}_{i}(\boldsymbol{x})}{\partial \boldsymbol{x}} \boldsymbol{G}(\boldsymbol{x})=\mathbf{0} .
$$

We now consider the I/O-linearization with time-varying constraints. The linearization is represented by the transformation (5), which contains the mapping $\boldsymbol{\varphi}(\boldsymbol{x}, t)$ for the states of the internal dynamics. Comparing (25) with (6) shows that

$$
\boldsymbol{\varphi}(\boldsymbol{x}, t)=\boldsymbol{\Psi}_{i}(\boldsymbol{x})
$$

fulfills (6) and is a suitable choice for the mapping of the internal states. As $\boldsymbol{\Psi}_{i}(\boldsymbol{x})$ is part of an invertible transformation (24), it provides an unambiguous mapping of the states and hence (5) with $\boldsymbol{\varphi}(\boldsymbol{x}, t)=\boldsymbol{\Psi}_{i}(\boldsymbol{x})$ is also an invertible coordinate transformation.

With this result, we are able to show that the stability properties of the internal dynamics for time-invariant constraints are preserved even for time-varying constraints.

Theorem 2: Consider the system (1) and the output function (2). Let Assumptions 3-5 hold. Let the internal dynamics resulting from I/O-linearization with time-invariant parameters $\boldsymbol{\eta}$ be stable in the sense of Lyapunov. Then, the internal dynamics, resulting from the I/O-linearization (7) with respect to each time-varying output function $i$ in (2), are stable in the sense of Lyapunov.

Proof: As for time-invariant output functions, the internal dynamics are assumed to be stable in the sense of Lyapunov, there exists a positive definite Lyapunov function $V_{\xi, i}\left(\boldsymbol{\xi}_{\text {int }, i}\right)$, which fulfills

$$
\begin{aligned}
V_{\xi, i}\left(\boldsymbol{\xi}_{\text {int }, \boldsymbol{i}}\right)>0 & \boldsymbol{\xi}_{\text {int }, \boldsymbol{i}} \neq \mathbf{0} \\
V_{\xi, i}\left(\boldsymbol{\xi}_{\text {int }, \boldsymbol{i}}\right)=0 & \boldsymbol{\xi}_{\text {int }, \boldsymbol{i}}=\mathbf{0} \\
\dot{V}_{\xi, i}\left(\boldsymbol{\xi}_{\text {int }, \boldsymbol{i}}, \boldsymbol{\xi}_{i}\right) \leq 0 & \boldsymbol{\xi}_{\text {int }, \boldsymbol{i}} \neq \mathbf{0}, \boldsymbol{\xi}_{i} \in \mathbb{R}^{r_{i}} \\
\dot{V}_{\xi, i}\left(\boldsymbol{\xi}_{\text {int }, \boldsymbol{i}}, \boldsymbol{\xi}_{i}\right)=0 & \boldsymbol{\xi}_{\text {int }, \boldsymbol{i}}=\mathbf{0}, \boldsymbol{\xi}_{i} \in \mathbb{R}^{r_{i}} .
\end{aligned}
$$

Choosing $V_{\xi, t, i}\left(\boldsymbol{\xi}_{\text {int }, \boldsymbol{i}}\right)=V_{\xi, i}\left(\boldsymbol{\xi}_{\text {int }, \boldsymbol{i}}\right)$ as Lyapunov function for the time-varying internal dynamics, its time derivative is given by

$$
\begin{aligned}
\dot{V}_{\xi, t, i}\left(\boldsymbol{\xi}_{\text {int }, \boldsymbol{i}}, \boldsymbol{\xi}_{i}, t\right) & =\frac{d V_{\xi, i}\left(\boldsymbol{\xi}_{\text {int }, \boldsymbol{i}}\right)}{d t} \\
& =\frac{\partial V_{\xi, i}\left(\boldsymbol{\xi}_{\text {int }, \boldsymbol{i}}\right)}{\partial \boldsymbol{\xi}_{\text {int }, \boldsymbol{i}}} \frac{d \boldsymbol{\xi}_{\text {int }, \boldsymbol{i}}}{d t} .
\end{aligned}
$$

With the transformation $\boldsymbol{\xi}_{\text {int }, i}=\Psi_{i}(\boldsymbol{x})$ from Proposition 1, the time derivative transforms into

$$
\begin{aligned}
\dot{V}_{\xi, t, i}\left(\boldsymbol{\xi}_{\mathrm{int}, \boldsymbol{i}}, \boldsymbol{\xi}_{i}, t\right) & =\frac{\partial V_{\xi, i}\left(\boldsymbol{\xi}_{\mathrm{int}, \boldsymbol{i}}\right)}{\partial \boldsymbol{\xi}_{\mathrm{int}, \boldsymbol{i}}} \frac{d \boldsymbol{\Psi}_{i}(\boldsymbol{x})}{d t} \\
& =\frac{\partial V_{\xi, i}\left(\boldsymbol{\xi}_{\mathrm{int}, \boldsymbol{i}}\right)}{\partial \boldsymbol{\xi}_{\mathrm{int}, \boldsymbol{i}}} \frac{\partial \boldsymbol{\Psi}_{i}(\boldsymbol{x})}{\partial \boldsymbol{x}} \frac{d \boldsymbol{x}}{d t} \\
& =\frac{\partial V_{\xi, i}\left(\boldsymbol{\xi}_{\mathrm{int}, \boldsymbol{i}}\right)}{\partial \boldsymbol{\xi}_{\mathrm{int}, \boldsymbol{i}}} \frac{\partial \boldsymbol{\Psi}_{i}(\boldsymbol{x})}{\partial \boldsymbol{x}}(\boldsymbol{f}(\boldsymbol{x})+\boldsymbol{G}(\boldsymbol{x}) \boldsymbol{u}) .
\end{aligned}
$$


Using (25) yields

$$
\dot{V}_{\xi, t, i}\left(\boldsymbol{\xi}_{\text {int }, i}, \boldsymbol{\xi}_{i}, t\right)=\frac{\partial V_{\xi, i}\left(\boldsymbol{\xi}_{\text {int }, i}\right)}{\partial \boldsymbol{\xi}_{\text {int }, i}} \frac{\partial \boldsymbol{\Psi}_{i}(\boldsymbol{x})}{\partial \boldsymbol{x}} \boldsymbol{f}(\boldsymbol{x}) .
$$

The Lyapunov function $V_{\xi, i}\left(\boldsymbol{\xi}_{\text {int }, i}\right)$ and the transformation $\boldsymbol{\Psi}_{i}(\boldsymbol{x})$ for the internal dynamics are equal to the time-invariant case, cf. Proposition 1. Therefore, the time derivative is equal to the time-invariant case (29)

$$
\dot{V}_{\xi, t, i}\left(\boldsymbol{\xi}_{\text {int }, i}, \boldsymbol{\xi}_{i}, t\right)=\dot{V}_{\xi, i}\left(\boldsymbol{\xi}_{\text {int }, i}, \boldsymbol{\xi}_{i}\right) \leq 0
$$

showing stability in the sense of Lyapunov.

With stable internal dynamics and a stabilizing nominal controller, in the following, the invariance properties of the invariance controlled system are examined.

\section{INVARIANCE}

Positive invariance of the controlled system is achieved, if the invariance controller assures that (18) is fulfilled. This requires the determination of suitable control parameters $\gamma_{i}$, which ensure that the corrective control input $\boldsymbol{u}_{\mathrm{c}}$ renders the controlled system invariant.

Theorem 3: Consider the system (1) and the output (2). Let Assumptions 1-4 hold. Let the matrix $\boldsymbol{A}_{\mathcal{K}_{\mathcal{I}}}(\boldsymbol{x})$ consist of the active constraints in $\mathcal{K}_{\mathcal{I}}$ fulfilling (21). Then, if there exists a

$$
\boldsymbol{\gamma}_{t}=\inf _{t \geq 0}\left(\sum_{k=0}^{r_{i}} \frac{r_{i} !}{\left(r_{i}-k\right) ! k !} \mathcal{L}_{\boldsymbol{f}}^{k}\left(\frac{\partial}{\partial t}\right)^{r_{i}-k} \boldsymbol{h}_{\mathcal{K}_{\mathcal{I}}}(\boldsymbol{x}, \boldsymbol{\eta})\right),
$$

with $\gamma_{t}>-\infty, \boldsymbol{u}_{\mathrm{c}}$ as determined by (22), renders the system positively controlled invariant with respect to the invariant set (15) for any choice of $z_{\mathrm{c}} \preccurlyeq \gamma$ fulfilling

$$
\gamma \preccurlyeq \gamma_{t} \quad \text { and } \quad \gamma \prec \mathbf{0}
$$

Proof: The corrective control input is given by $\boldsymbol{u}_{\mathrm{c}}=\boldsymbol{A}_{\mathcal{K}_{\mathcal{I}}}^{+}\left(\boldsymbol{z}_{\mathrm{c}}-\boldsymbol{b}_{\mathcal{K}_{\mathcal{I}}}\left(\boldsymbol{x}, \boldsymbol{\eta}, \ldots, \boldsymbol{\eta}^{\left(r_{i}\right)}\right)\right)+\left(\boldsymbol{I}-\boldsymbol{A}_{\mathcal{K}_{\mathcal{I}}}^{+} \boldsymbol{A}_{\mathcal{K}_{\mathcal{I}}}\right) \boldsymbol{u}_{\mathrm{no}}$ with $\boldsymbol{A}_{\mathcal{K}_{\mathcal{I}}}=\boldsymbol{A}_{\mathcal{K}_{\mathcal{I}}}(\boldsymbol{x}, \boldsymbol{\eta})$. The matrix $\boldsymbol{I}-\boldsymbol{A}_{\mathcal{K}_{\mathcal{I}}}^{+} \boldsymbol{A}_{\mathcal{K}_{\mathcal{I}}}$ represents a projection of the nominal control input $\boldsymbol{u}_{\text {no }}$ into the null-space of the active constraints and each column of $\boldsymbol{A}_{\mathcal{K}_{\mathcal{I}}}^{+}$ is in increasing direction of one active constraint with no influence on the other active constraints [14]. For active constraints, the pseudo control input is set to $z_{\mathrm{c}} \preccurlyeq \gamma(23)$. Therefore, a sufficient condition for invariance with timevarying constraints is given by

$$
\boldsymbol{\gamma}-\boldsymbol{b}_{\mathcal{K}_{\mathcal{I}}}\left(\boldsymbol{x}, \boldsymbol{\eta}, \ldots, \boldsymbol{\eta}^{\left(r_{i}\right)}\right) \preccurlyeq 0 .
$$

Using (7) and (12), the condition (34) transforms into

$$
\begin{aligned}
& \gamma \preccurlyeq \overline{\mathcal{L}}_{\boldsymbol{f}}^{r_{i}} \boldsymbol{h}_{\mathcal{K}_{\mathcal{I}}}(\boldsymbol{x}, \boldsymbol{\eta}) \\
& \gamma \preccurlyeq\left(\frac{\partial}{\partial t}+\mathcal{L}_{\boldsymbol{f}}\right)^{r_{i}} \boldsymbol{h}_{\mathcal{K}_{\mathcal{I}}}(\boldsymbol{x}, \boldsymbol{\eta}) .
\end{aligned}
$$

Applying the binomial theorem yields

$$
\boldsymbol{\gamma} \preccurlyeq \sum_{k=0}^{r_{i}} \frac{r_{i} !}{\left(r_{i}-k\right) ! k !} \mathcal{L}_{f}^{k}\left(\frac{\partial}{\partial t}\right)^{r_{i}-k} \boldsymbol{h}_{\mathcal{K}_{\mathcal{I}}}(\boldsymbol{x}, \boldsymbol{\eta}) .
$$

Keep in mind that the partial derivative with respect to time of the output function is given by

$$
\frac{\partial \boldsymbol{h}_{\mathcal{K}_{\mathcal{I}}}(\boldsymbol{x}, \boldsymbol{\eta})}{\partial t}=\frac{\partial \boldsymbol{h}_{\mathcal{K}_{\mathcal{I}}}(\boldsymbol{x}, \boldsymbol{\eta})}{\partial \boldsymbol{\eta}} \frac{d \boldsymbol{\eta}}{d t} .
$$

If there exists a constant lower bound

$$
\boldsymbol{\gamma}_{t}=\inf _{t}\left(\sum_{k=0}^{r_{i}} \frac{r_{i} !}{\left(r_{i}-k\right) ! k !} \mathcal{L}_{\boldsymbol{f}}^{k}\left(\frac{\partial}{\partial t}\right)^{r_{i}-k} \boldsymbol{h}_{\mathcal{K}_{\mathcal{I}}}(\boldsymbol{x}, \boldsymbol{\eta})\right) \text {, }
$$

the choice of $\gamma \preccurlyeq \gamma_{t}$ fulfills

$$
\boldsymbol{z}_{\mathrm{c}} \preccurlyeq \boldsymbol{\gamma} \preccurlyeq \boldsymbol{\gamma}_{t} \preccurlyeq \boldsymbol{b}_{\mathcal{K}_{\mathcal{I}}}\left(\boldsymbol{x}, \boldsymbol{\eta}, \ldots, \boldsymbol{\eta}^{\left(r_{i}\right)}\right)
$$

and therefore renders the system positively controlled invariant, cf. (34). Additionally, corrective control may only ensure invariance, if it decreases the value of the invariance function. The invariance function decreases if the states of the integrator chain decrease, which is only possible for a non-positive input of the integrator chain $z_{\mathrm{c}} \preccurlyeq \gamma$. Therefore

$$
z_{\mathrm{c}} \preccurlyeq \gamma \prec \mathbf{0}
$$

has to be fulfilled element-wise in addition to (34).

\section{NumERICAL EXAMPLE}

The numerical example uses the angular dynamics of a rigid inverted pendulum on a cart to illustrated the capabilities of the derived approach.

\section{A. Setup}

The angular dynamics of the pendulum are given by

$$
\begin{aligned}
\dot{\boldsymbol{x}} & =\boldsymbol{f}(\boldsymbol{x})+g(\boldsymbol{x}) u \\
\boldsymbol{f}(\boldsymbol{x}) & =\left[\begin{array}{c}
x_{2} \\
\frac{g}{l} \sin \left(x_{1}\right)-\frac{m \cos \left(x_{1}\right) \sin \left(x_{1}\right)\left(l x_{2}^{2}+g \cos \left(x_{1}\right)\right)}{l\left(m \sin ^{2}\left(x_{1}\right)+M\right)}
\end{array}\right] \\
g(\boldsymbol{x}) & =\frac{\cos \left(x_{1}\right)}{l\left(m \sin ^{2}\left(x_{1}\right)+M\right)},
\end{aligned}
$$

with the masses of the pendulum $m$ and the cart $M$, the length $l$, the gravity constant $g$ and the angle $x_{1}$ to the upright position. Nominal control is determined by the state feedback

$$
u_{\mathrm{no}}=-k_{P} x_{1}-k_{D} x_{2},
$$

which keeps the pendulum in the upright position. The timevarying constraint is given by

$$
h(\boldsymbol{x}, t)=x_{2}-\left(\frac{3}{2} x_{2, \mathrm{~b}}-\frac{1}{2} x_{2, \mathrm{~b}} f_{\mathrm{b}}(t)\right),
$$

with a constant minimum bound value $x_{2, \mathrm{~b}}$ and the function

$$
\left.f_{\mathrm{b}}(t)\right)= \begin{cases}\cos \left(\frac{2 \pi t}{T}\right) & \text { for } 0 \leq t \leq T \\ 1 & \text { else }\end{cases}
$$

with the constant time interval $T$. Derivation with respect to time yields the relative degree $r=1$, which is well-defined for $x_{1} \neq \pm \frac{\pi}{2}+k, k \in \mathbb{Z}$, as well as the I/O-linearization with $\boldsymbol{a}^{\boldsymbol{\top}}=g(\boldsymbol{x})$ and $b=f_{2}(\boldsymbol{x})-\dot{h}(\boldsymbol{x}, t)$. The invariance function is given by $\Phi(\boldsymbol{x}, t, \gamma)=h(\boldsymbol{x}, t)$ according to (14). and corrective control is determined using (22)-(23).

The implementation in Matlab/Simulink uses the parameters in Table I. The solution is determined by an Euler solver with a step size of $1 \cdot 10^{-5} \mathrm{~s}$. The results are compared to the unconstrained system under nominal control. 
TABLE I: Model parameters

\begin{tabular}{lll}
\hline System parameters & $\boldsymbol{x}(0)$ & {$\left[-5 \frac{\pi}{180} \mathrm{rad}, 0 \mathrm{rad} / \mathrm{s}\right]^{\top}$} \\
& $g$ & $9.81 \mathrm{~m} / \mathrm{s}^{2}$ \\
& $l$ & $0.2 \mathrm{~m}$ \\
& $m$ & $0.1 \mathrm{~kg}$ \\
& $M$ & $1 \mathrm{~kg}$ \\
Constraint & $x_{2, \mathrm{~b}}$ & $1 \frac{\pi}{180} \mathrm{rad} / \mathrm{s}$ \\
Nominal control & $T$ & $2.5 \mathrm{~s}$ \\
& $k_{P}$ & $10 \mathrm{~N}$ \\
Invariance control & $k_{D}$ & $1 \mathrm{~N} \mathrm{~s}$ \\
& $\gamma$ & $-0.11 / \mathrm{s}$
\end{tabular}

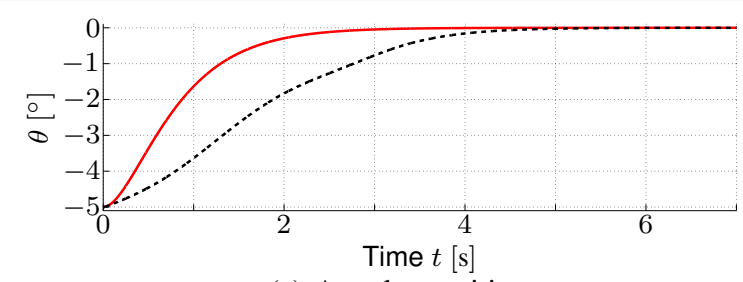

(a) Angular position

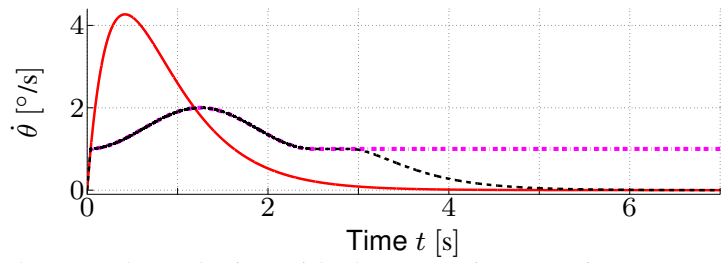

(b) Angular velocity with the -..- time-varying constraint

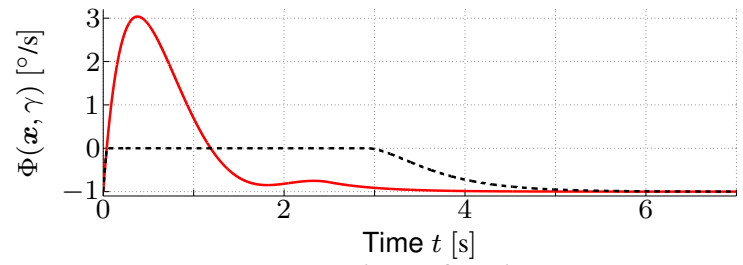

(c) Invariance function

Fig. 2: Results for the controlled pendulum - without constraint and - - - with constraint enforced by invariance control.

\section{B. Results}

The results of the simulation are depicted in Fig. 2. Initially, the pendulum deviates from the upright position as depicted in Fig. 2a. As the velocity is initially below the constraint, cf. Fig. 2b, the invariance function in Fig. 2c has a negative value and nominal control is applied, which results in the same behavior with and without constraints. Once the constraint is reached, invariance control enforces the time-varying constraint which is observed in Fig. $2 b$ and $2 \mathrm{c}$ as the velocity is never higher then the constraint and the invariance function has a non-positive value. The pendulum under nominal control, in contrast, violates the constraint by over $3 \%$, which may be observed in the invariance function. Naturally, the unconstrained pendulum reaches the desired upright position faster as depicted in Fig. 2a. However, the invariance controlled pendulum also reaches the desired position eventually, which is an indication for the preservation of the nominal stability property and stable internal dynamics.

\section{CONCLUSION}

In this work, a novel approach for invariance control with time-varying constraints is introduced. A control framework is presented, allowing for constraints with time-varying parameters and preserving the stability of the nominally controlled system. Additionally, the limits on the system, which are imposed by the constraints, are met, rendering the system positively controlled invariant with respect to the time-varying boundaries, if the parameter variation is bounded with continuous and bounded derivatives. A numerical example illustrates the stability and the invariance properties.

\section{ACKNOWLEDGEMENTS}

The research leading to these results has received funding from the European Union Seventh Framework Programme FP7/2007-2013 within the ERC Starting Grant Control based on Human Models (con-humo) under grant agreement no. 337654 .

\section{REFERENCES}

[1] D. Mayne, J. Rawlings, C. Rao, and P. Scokaert, "Constrained model predictive control: Stability and optimality," Automatica, vol. 36, no. 6 , pp. 789-814, 2000.

[2] E. Gilbert and I. Kolmanovsky, "Nonlinear tracking control in the presence of state and control constraints: a generalized reference governor," Automatica, vol. 38, no. 12, pp. 2063-2073, 2002.

[3] S. Prajna and A. Rantzer, "Convex Programs for Temporal Verification of Nonlinear Dynamical Systems," SIAM Journal on Control and Optimization, vol. 46, no. 3, pp. 999-1021, 2007.

[4] A. Ames, J. Grizzle, and P. Tabuada, "Control Barrier Function based Quadratic Programs with Application to Adaptive Cruise Control," in IEEE 53rd Annual Conference on Decision and Control (CDC), Dec 2014, pp. 6271-6278.

[5] J. Wolff and M. Buss, "Invariance Control Design for Nonlinear Control Affine Systems under Hard State Constraints," in NOLCOS'2004 Symposium on Nonlinear Control Systems, Stuttgart, Germany, Sept. 2004, pp. 711-716.

[6] M. Scheint, J. Wolff, and M. Buss, "Invariance Control in Robotic Applications: Trajectory Supervision and Haptic Rendering," in American Control Conference (ACC), Seattle, USA, 2008, pp. 1436-1442.

[7] J. Mareczek, M. Buss, and G. Schmidt, "Sufficient Conditions for Invariance Control of a Class of Nonlinear Systems," in 39th IEEE Conference on Decision and Control, Sydney, Australia, Dec. 2000, pp. 1436-1442

[8] J. Wolff and M. Buss, "Invariance Control Design for Constrained Nonlinear Systems," in 16th IFAC World Congress, Prague, Czech Republic, July 2005

[9] S. Palanki and C. Kravaris, "Controller synthesis for time-varying systems by input/output linearization," Computers \& Chemical Engineering, vol. 21, no. 8, pp. 891 - 903, 1997.

[10] K. Sekiguchi and M. Sampei, "Change of controller based on partial feedback linearization with time-varying function," in IEEE 51st Annual Conference on Decision and Control (CDC), 2012, pp. $3557-$ 3563

[11] A. Isidori, Nonlinear Control Systems, 3rd ed. Springer-Verlag, Berlin, 1995

[12] S. P. Boyd, Convex Optimization, L. Vandenberghe, Ed. Cambridge University Press, 2004.

[13] H. Khalil, Nonlinear Systems, 3rd ed. Prentice Hall, 1996.

[14] M. Kimmel and S. Hirche, "Invariance Control with Chattering Reduction," in IEEE 53rd Annual Conference on Decision and Control $(C D C), 2014$. 